\title{
Desarrollo de soft skills una alternativa a la escasez de talento humano
}

\section{Development of soft skills an alternative to the shortage of human talent}

\author{
María D. Tito Maya \\ Universidad de Especialidades Espíritu Santo, Ecuador
}

Bill Serrano Orellana

Universidad Técnica de Machala, Ecuador

Autor para correspondencia: bjserrano@utmachala.edu.ec

Fecha de recepción: 12 de Septiembre de 2016 - Fecha de aceptación: 01 de Noviembre de 2016

\section{Resumen}

La época actual, caracterizada por la instauración de una sociedad basada en la información, el cambio acelerado, la globalización, y la tecnología; ha traído como consecuencia una nueva configuración de los empleos. El objetivo de este artículo es dar a conocer mediante la exposición de distintas investigaciones, que los conocimientos o destrezas técnicos, mecánicos y prácticos conocidos como hard skills ya no son suficientes para tener éxito. Los empresarios de hoy, dan por sentado que se tiene suficiente preparación técnica e intelectual, para concentrarse en contratar personas que cuenten con habilidades blandas o soft skills. Si bien esto es considerado como una ventaja competitiva en las empresas, la comunidad educativa brinda poca importancia a la enseñanza de éstas habilidades. Eso explica de cierta forma, la escasez de talentos que existe ahora en el mercado laboral. Los resultados de este trabajo, permitirán conocer un perfil de competencias blandas deseable para todos los empleados en esta nueva era; y diferentes herramientas para desarrollar estas habilidades.

Palabras claves: contrato psicológico; compromiso organizacional; liderazgo

\begin{abstract}
The present era, characterized by the establishment of the society based on information, rapid change, globalization, and technology; it has resulted in a new set of jobs. The aim of this paper is to show, by exposing different investigations, which acquaintances technical knowledge, and practical skills such as mechanical hard skills are no longer enough to succeed. Employers today, assume that you have sufficient technical and intellectual preparation, to concentrate on hiring people that have soft skills. While this is considered a competitive advantage in business, education community provides little importance to the teaching of these skills. That explains a certain way, the talent shortage that now exists in the labor market. The results of this work will allow to know a desirable soft skills profile required by all employees in this new era; and different tools for training in these skills.
\end{abstract}

Key words: soft skills; education; development; human talent 


\section{Introducción}

La época actual, caracterizada por el rápido intercambio de información y difusión de tecnología, el cambio acelerado y la globalización, ha configurado nuevos mercados laborales en los que se pone de manifiesto una escasez de perfiles competitivos (Manpower, 2013; Vargas; 2006). ¿Qué se entiende por talento humano? A lo largo de la historia han existido varias interpretaciones sobre este concepto, la real academia de la lengua española lo define como la inteligencia y aptitud de una persona para determinada ocupación. (Diccionario de la Lengua Española, 2001). Martha Alles (2008) une a los conceptos de talento y competencias como sinónimos, de esta forma demostrar talento para una determinada ocupación implica la posibilidad de desglosarlo en competencias que pueden ser medidas y desarrolladas: "Las competencias son características de la personalidad devenidas en comportamientos que generan un desempeño exitoso/superior en un puesto de trabajo" (Alles, 2008, p.29).

Las competencias pueden ser clasificadas como: blandas (características de personalidad, difíciles de desarrollar, y de amplio alcance) y duras (conocimientos técnicos, prácticos, mecánicos, específicos para una tarea o actividad) (Alles, 2007). En inglés se conocen como soft and hard skills.

Un estudio desarrollado por Manpower (2013) sobre la escasez de talento humano en el mercado, afirma que una de las principales dificultades para encontrar candidatos es la falta de habilidades de empleabilidad o "soft skills":

Uno de cada cinco directivos (19\%) afirma que los candidatos carecen de las competencias de empleabilidad (las llamadas habilidades sociales o soft skills) requeridas. Los directivos han identificado una serie de déficits en materia de habilidades sociales, entre los que se incluyen el entusiasmo/ motivación (5\%), las habilidades de trato interpersonal (4\%), la profesionalidad, es decir, el cuidado por el aspecto, la puntualidad, etc. (4\%), y la flexibilidad y adaptabilidad (4\%). (Manpower, 2013, p. 8)

De acuerdo a la Organización Internacional del Trabajo OIT la tasa de desempleo juvenil en el 2013 fue del 14,5\% en Latinoamérica. Contrariamente a esta información, Flores (2014) indica que el $39 \%$ de las empresas de la región tuvieron dificultad para cubrir vacantes en ese mismo período, por lo que señalan que existe una escasez de talentos en el mercado. La autora, menciona que la brecha entre la oferta y demanda de talentos se debe a muchos factores, entre los que destaca el hecho de que los programas educativos presentan poca articulación con las estrategias empresariales:

No se prepara a los jóvenes en las habilidades y las competencias necesarias para un trabajo formal, el aprendizaje suele ser de carácter memorístico y centrado en el maestro. Mientras que el $72 \%$ de unidades educativas señala que sus recién graduados están listos para trabajar, el $42 \%$ y el $45 \%$ de graduados y empleadores señalan que no lo están respectivamente (Flores, p.36).

Así pues, se considera que el desarrollo de competencias y del talento humano en general, integrará las agendas de gobiernos e instituciones del mundo entero y representará uno de los 
grandes desafíos de los años venideros (Alles, 2008). En Ecuador, conscientes que la globalización es un fenómeno que afecta a todos los países del mundo, también será indispensable que se identifique la necesidad de desarrollar talento con visión, iniciativa, y conocimiento multicultural; para que se formen líderes capaces de responder estratégicamente a contextos mundiales (Tola, 2012). Para hacerlo es necesario replantear los contenidos de la educación que se imparte en todos los niveles (escuelas, colegios, universidades) diseñando currículos de calidad, más flexibles, que sean capaces de ajustarse a las nuevas demandas del mercado, y que se encuentren focalizados en el desarrollo humano y la ampliación de oportunidades para el mejoramiento de la calidad de vida de las personas. Esto también implicaría empezar a educar y cultivar habilidades blandas (Bermúdez, Montaño, \& Santillan, 2011).

Este trabajo se centrará en el análisis de las soft skills, debido a que éstas son las más difíciles de desarrollar y las que generan un desempeño superior en el puesto de trabajo. De acuerdo a Puga (2008) las competencias más demandadas por el mercado laboral actual se vinculan más con capacidades mentales y/o personales -llamadas habilidades blandas- que con destrezas mecánicas o prácticas. Además, porque en el mundo competitivo de hoy no basta con pensar que las "habilidades duras" por sí solas son suficientes para el éxito en el trabajo. Los conocimientos, técnicas y destrezas son el requisito mínimo para desempeñarse en una posición, sin embargo estas "habilidades mínimas aceptables" están siendo reemplazadas por estándares cada vez más altos. Precisamente entre estos estándares, emergen lo que muchos llaman "habilidades blandas" (Lazarus, 2013).

Por lo tanto, esta investigación dará a conocer un perfil de competencias blandas deseable para cualquier colaborador, independientemente del campo profesional o tipo de industria en el que se encuentre. Entender las metodologías utilizadas para desarrollar estas habilidades desde las aulas; y así mejorar las oportunidades de empleabilidad de los estudiantes.

Además, al ser un trabajo centrado en entender cómo desarrollar al máximo las capacidades humanas para el empleo, se evidencia una estrecha relación con los objetivos tres y cuatro del Plan Nacional del Buen Vivir - PNBV (2013-2017): 3.- "Mejorar la calidad de vida de la población" y 4.- "Fortalecer las capacidades y potencialidades de la ciudadanía". En otras palabras, al mejorar el talento humano desde las aulas perfeccionando sus habilidades de empleabilidad, se estaría promoviendo la reciprocidad entre la educación y el sector productivo, lo cual permitiría elevar la calidad de vida de la sociedad en general.

\section{Marco Teórico}

\section{Origen de las competencias - Definición de competencias blandas o soft skills}

Se ha hecho la transición de la era de industrialización, a la era de producción industrial y servicios, en donde se instauró la sociedad basada en el conocimiento y la información. Todo esto, sumado a la globalización, las reformas políticas, económicas y sociales, y el uso de tecnología, ha tenido una enorme repercusión en la configuración de los empleos y por ende en la demanda de las habilidades o competencias que se requieren de los trabajadores. Este nuevo contexto, ha propiciado el que se deje de lado lo que antes era importante - como la medición de 
tiempos de trabajo, operaciones y movimientos, siendo ahora el eje central las competencias claves o transversales- en función de las motivaciones y capacidades del trabajador (Vargas, 2006).

El significado de las competencias tiene su origen en los estudios realizados por David McClelland (1987) en donde analiza cuáles son los motivos que orientan, energizan, y determinan los comportamientos de las personas. Este autor hizo importantes avances al respecto definiendo 3 sistemas motivacionales: Motivación por el logro, motivación por el poder, motivación por la afiliación (Alles, 2007). Más adelante, y siguiendo los estudios de McClelland, Spencer y Spencer (1993) señalan que la competencia es una característica en el individuo que está causalmente relacionada con un estándar de efectividad y/o con un desempeño superior en una situación de trabajo. Indican también que son formas de comportamiento o de pensar que se presentan en distintas situaciones y que son estables en el tiempo. De esta forma agrupan las competencias en distintas categorías: Motivación, características físicas o respuestas consistentes a una situación, concepto de uno mismo, conocimiento y habilidad (Spencer y Spencer, 1993; citados en Alles, 2008). De acuerdo a estos autores la parte visible y por tanto más fácil de identificar y de desarrollar son los conocimientos y las destrezas; y la parte no visible, y a su vez más difícil de desarrollar e identificar son: el concepto de uno mismo y los rasgos de personalidad.

Blanco (2009) indica que existen tres enfoques para entender el significado de las competencias: el primero, concibe las competencias como la capacidad de ejecutar las tareas, por ejemplo, aplicar cuidados auxiliares de enfermería al paciente. El segundo, se concentra en atributos personales como "autoestima", "integridad". Y existe un tercer enfoque que es holístico que incluye los otros dos por ejemplo: la competencia se concibe como una compleja estructura de atributos necesarios para el desempeño de situaciones específicas. La autora señala que por atributos se entienden los conocimientos, actitudes, valores, y habilidades, que se ponen en juego para actuar en una situación específica.

Otra clasificación de competencias son las llamadas competencias transversales o claves, según Blanco (2009) las competencias transversales, claves o de empleabilidad suelen entenderse como sinónimos. Mertens (1996) denomina a las competencias claves como competencias de empleabilidad puesto que son necesarias para conseguir un empleo, permanecer en él o encontrar uno nuevo. Advierte que son "transversales" debido a que es el total de la población la que debe poseerlas, ya sea por razones de equidad (para evitar la marginación) como por razones de productividad (porque así los trabajadores pueden continuar aprendiendo, afrontar cambios y oportunidades). Blanco (2009) manifiesta que las competencias de empleabilidad son principalmente de tipo social (habilidades de comunicación, trabajo en equipo). En el ámbito de estas competencias sociales se ha desarrollado también el concepto de "competencias blandas". Por lo tanto, según ambos autores las competencias blandas son equivalentes a las competencias de empleabilidad, claves y transversales y son especialmente de tipo social.

Los autores Geana W., Leane B., y Bonnie J. (2010), señalan que las competencias blandas describen los atributos personales como habilidades de trabajo en equipo, habilidades de comunicación, la ética, habilidades de gestión del tiempo, y el aprecio por la diversidad. En el siglo XXI, las habilidades blandas son importantes en todos los sectores. El Diccionario Collins 
(2014) define el término habilidades blandas como "cualidades deseables para ciertas formas de empleo que no dependen de los conocimientos adquiridos: incluyen el sentido común, la capacidad de tratar con la gente, y una actitud positiva y flexible"

Hewitt (2006) describe las habilidades blandas como esencialmente de tipo interpersonal -habilidades no técnicas, intangibles-, en la personalidad que determinan la fuerza de un individuo como un líder, oyente, negociador y mediador de conflictos. Mientras que las habilidades duras son conocimientos relacionados a la educación y nivel de experiencia, que permiten el manejo de datos, equipos y sistemas (Laker \& Powell, 2011). Ejemplos de habilidades duras incluyen: escritura, matemáticas, estadística, finanzas, química, biología, la capacidad de utilizar los programas de software, entre otros; (Han, 2014).

Martha Alles (2008) presenta un enfoque más práctico para entender el concepto de competencias blandas. Como se ha visto, muchos autores agrupan a los conocimientos como parte de las competencias y además incluyen a las habilidades y destrezas. La autora separa a las habilidades y destrezas, de las competencias, -en la medida en que son fáciles de detectar y desarrollar y sobre todo porque por sí mismas no se relacionan con el desempeño superior en el puesto de trabajo-, para enfocarse en los rasgos de personalidad que devienen en comportamientos que permiten un desempeño superior. Es decir, las competencias serían aquellos comportamientos que son observables y medibles y que se relacionan con un desempeño superior en una situación específica. Aquí un ejemplo de la diferencia de estos 3 conceptos:

Tabla \# 1: Ejemplos de Conocimientos Técnicos, Destrezas, y Habilidades Blandas.

\begin{tabular}{|c|c|c|}
\hline $\begin{array}{l}\text { Conocimientos Técnicos, = Habilidades Duras o } \\
\text { Hard Skills }\end{array}$ & $\begin{array}{l}\text { Habilidades, } \\
\text { Destrezas }\end{array}$ & $\begin{array}{l}\text { Competencias }=S o f t \\
\text { Skills }\end{array}$ \\
\hline Matemáticas aplicadas & Manejo de vehículos & Adaptabilidad \\
\hline Estadística & $\begin{array}{l}\text { Manejo de } \\
\text { ordenadores }\end{array}$ & Aprendizaje continuo \\
\hline Contabilidad General & $\begin{array}{l}\text { Preparación de } \\
\text { comidas }\end{array}$ & Autocontrol \\
\hline Costos & Reparación de objetos & Autonomía \\
\hline Idiomas & $\begin{array}{l}\text { Resistencia al } \\
\text { cansancio }\end{array}$ & Autodirección \\
\hline
\end{tabular}

Nota. Por M. Alles, 2008, Desarrollo del talento humano basado en competencias, p.42-43

Por lo tanto, las competencias duras son el expertise técnico y los conocimientos necesarios para ejecutar un trabajo. Mientras que las competencias blandas son las cualidades intrapersonales y de relacionamiento. Los ejecutivos de negocios consideran las competencias blandas como un atributo muy importante para los postulantes a una oferta de trabajo. Los empleadores esperan que los trabajadores cuenten con un conjunto sólido de competencias blandas al igual que técnicas (Robles, 2012).

Perreault (2004) define el término "soft skills" como las cualidades o atributos personales o nivel de compromiso de una persona que la distingue de los demás aunque éstos posean similares habilidades y tipo de experiencia. James y James (2004) concuerdan en que las "habilidades blandas" son un conjunto de cualidades personales como: comunicación, liderazgo, servicio al cliente, resolución de problemas, trabajo en equipo, que permiten a los individuos 
tener éxito en el mercado laboral y promover su carrera. Schulz (2008) define a las competencias blandas como aquellas características de personalidad y habilidades interpersonales más demandadas por un empleador cuando está buscando personal para ejecutar cualquier tipo de trabajo.

En virtud de lo descrito hasta aquí, existen diferentes clasificaciones de las competencias. Este trabajo se enfocará en las habilidades blandas (soft skills) que de acuerdo a Blanco (2009) pueden ser tratadas como sinónimo de competencias de empleabilidad, claves o transversales. Se separarán de los conceptos de conocimientos y destrezas, debido a que solo las habilidades blandas están asociadas con presentar un desempeño superior en el trabajo y son éstas las que constituyen una ventaja diferencial en el mercado laboral.

\section{Importancia de las competencias blandas}

En la actualidad los mercados de trabajo se rigen por nuevas reglas de juego. Estas nuevas reglas guardan poca relación con lo que antes era importante en la escuela, donde se daba mayor peso a la formación académica. Ahora los mercados dan por sentado que se cuenta con suficiente preparación técnica e intelectual, para concentrarse en la demanda de cualidades personales como la empatía, adaptabilidad, comunicación (Alles, 2007).

El trabajo que realizan los empleados de hoy, se compone de muchas menos tareas repetitivas e individuales. Este nuevo contexto, espera colaboradores que sean más autónomos y capaces de trabajar con y a través de otros. Lo que ha ocasionado un aplanamiento de la jerarquía de la organización tradicional donde las habilidades blandas se vuelven esenciales para todos los empleados, no solo para aquellos que ocupan un rol de supervisión (Brungardt, 2011).

La mejor forma de preparar la fuerza laboral del mañana se basa no solamente en el desarrollo de habilidades técnicas sino en el fortalecimiento de habilidades intrapersonales y de interacción con otros. Ahora más que nunca, ser técnicamente competente no es suficiente. Para tener éxito en un entorno de trabajo globalizado y diverso, los estudiantes deben desarrollar las relaciones humanas, la autogestión, y habilidades de eficacia en el lugar de trabajo. Siendo así, los alumnos deben entender el beneficio de las habilidades blandas en sus carreras y vidas personales. (Policies Commission for Business and Economic Education [PCBEE], 2000)

Sutton (2002) señala que las habilidades blandas son tan importantes que los empleadores las reconocen como el "diferenciador número uno" para quienes aspiran a un puesto de trabajo sin importar el tipo de industria o profesión. Glenn (2008) adicionalmente menciona que contratar individuos que cuenten con "habilidades blandas" es una estrategia de gran importancia para las empresas de alto rendimiento que deseen construir y mantener una ventaja competitiva. Wilhelm (2004) afirmó que los empleadores valoran altamente las habilidades blandas como factor de éxito para ingresar a una empresa desde los primeros niveles jerárquicos.

Una fuerza de trabajo que cuente con ciertas competencias claves fortalecerá la capacidad de su empleador para competir. A su vez, estas competencias brindan al trabajador mayores oportunidades de desarrollo y una mejor calidad de vida. A los trabajadores con buenas 
habilidades claves les resulta más fácil adquirir destrezas más sofisticadas, para así conseguir mejores empleos y salarios más altos (Carnevale, Gainer, \& Meltzer, 1990).

\section{Definición de un perfil de competencias blandas transversal (independiente de las profesiones e industrias)}

Para Blanco (2009) las competencias generales que todos los graduados deben poseer independientemente de su carrera para desempeñarse con éxito en el campo laboral, son: trabajo en equipo, planificación, comunicación, bilingüismo, búsqueda y procesamiento de información, análisis y síntesis.

Schulz (2008) determina que entre los requisitos más importantes que deben poseer los graduados están las habilidades de comunicación, el pensamiento crítico o estructurado y la creatividad.

Las competencias más demandadas por el mundo empresarial actual y que son transversales al tipo de empresa o entorno, de acuerdo a diferentes revisiones teóricas e investigaciones son cinco: orientación al conocimiento; liderazgo; habilidad de comunicación; valores éticos y habilidad para trabajar en equipo (Puga \&Martínez, 2008).

Employers Value Communication and Interpersonal Skills - EVCIS (2004) sugiere que una persona que se comunica efectivamente, se lleva bien con los demás, sabe trabajar en equipo y posee una sólida ética de trabajo, puede ser considerado como alguien con un completo set de habilidades blandas.

Mitchell, Skinner, y White (2010) realizaron un estudio en el que se encuestó a diferentes centros educativos de Alabama con el propósito de dar a conocer cuáles son las competencias blandas más importantes para tener éxito en el mercado laboral del siglo XXI. Los resultados de la encuesta revelaron la importancia de las siguientes habilidades blandas en orden descendente: la comunicación en general, ética, gestión del tiempo / organización, comunicación escrita, trabajo en equipo, etiqueta de negocios, diversidad, servicio al cliente, resolución de problemas / pensamiento crítico, comunicación oral, y liderazgo.

Por su parte, un estudio realizado por la Universidad de Kentucky USA en el 2011 en el cual se solicitó a distintas escuelas de negocios que enlisten las 10 habilidades blandas necesarias para triunfar en el mercado laboral actual, reveló que la integridad, comunicación, cortesía, responsabilidad, habilidades interpersonales, profesionalismo, actitud positiva, trabajo en equipo, flexibilidad, y ética de trabajo, en ese orden, son las más importantes para tener éxito en el mercado laboral (Robles, 2012).

El 96\% de 330 ejecutivos encuestados por la Universidad de Phoenix en 2005 señalaron que la comunicación y las habilidades interpersonales son las cualidades más valoradas que deben poseer los empleados, mientras que el $95 \%$ calificó la capacidad de aprendizaje y el deseo de crecer; seguido por la colaboración y el trabajo en equipo que fueron marcados por el 93\%, y la solución creativa de problemas por el 92\% (Cline, 2005). 
Según Ellis, Kisling \& Hackworth (2014) una encuesta realizada por South Carolina Chamber of Commerce (SCCC) a diferentes empresas en el 2000 y 2006, sobre las competencias que deben tener los empleados para tener éxito en el trabajo dio como resultado 37 habilidades de las cuales 23 fueron soft skills, a continuación se enlistan cuales: Administrar el dinero, administrar los recursos humanos, interpretar y transmitir información, atender a los clientes y consumidores, participar como miembro de un equipo, trabajar con la diversidad, ejercer liderazgo, negociar una decisión, enseñar a los demás, escuchar, expresarse oralmente, resolver problemas, razonar, capacidad de aprendizaje, tomar de decisiones, creatividad, análisis, integridad, responsabilidad, autocontrol, habilidades sociales y autoestima.

De acuerdo con los resultados de una encuesta publicada por American Management Association (AMA), los ejecutivos aseguran que el conocimiento y las habilidades típicas en las áreas de lectura, escritura y aritmética (las tres R en inglés: reading, writing, and arithmetic) ya no son suficientes para los directivos. Para asegurar el éxito en la fuerza de trabajo del futuro, las tres R necesitan ser fusionadas con otras cuatro habilidades (las cuatro C): 1) el pensamiento crítico y habilidades para resolver problemas, 2) habilidades de comunicación, 3) habilidades de colaboración, y, 4) creatividad e innovación (Hales, 2012).

Profundizando un poco más en estas competencias, las habilidades de comunicación si bien tienen un amplio espectro, podrían resumirse de la siguiente forma: dominio del lenguaje oral y escrito, el lenguaje corporal, y habilidades de presentación para poder comercializar las propias ideas y a uno mismo. Las habilidades de comunicación son una supra habilidad necesaria para generar resultados en cualquier nivel de la organización y promover la carrera profesional de una persona. Además éstas se encuentran ligadas a las habilidades de interacción social y manejo de conflictos que son indispensables en el medio laboral para desempeñarse exitosamente (Schulz, 2008; Puga \& Martinez, 2008).

La capacidad de aprendizaje y el pensamiento crítico, son indispensables en este entorno globalizado, cambiante y de fácil acceso a la información. Ahora es imprescindible contar con colaboradores que tengan la capacidad de recopilar, diseminar y generar conocimiento para su propio desarrollo y para el de la empresa.

El trabajo en equipo que incluye las habilidades de interacción y cooperación, ha demostrado ser clave en las organizaciones, puesto que genera una filosofía de esfuerzo y logros compartidos, y un compromiso pactado con la colectividad antes que en lo individual, lo que genera mejores resultados en el largo plazo (Puga \& Martinez, 2008). Por otro lado, la creatividad se traduce como aquella capacidad que permite pensar más allá de lo establecido e innovar. Ésta no debe asociarse como algo propio de las carreras artísticas sino como un aspecto elemental para cualquier profesión. En el campo de los negocios una nueva idea puede resultar en un nuevo producto, proceso o procedimiento, que solucione algún problema y que se traduzca en mayores ingresos para la compañía. La creatividad es un recurso clave en organizaciones que están afrontando cambios o requieren consolidar ventajas competitivas (Codina, 2005).

En cuanto a la ética, se ha demostrado que la carencia de ésta puede ocasionar el quiebre de grandes organizaciones. Casos como los de Enron Corporation y Arthur Andersen, son una 
muestra de esto. ${ }^{1}$ Cuando la ética no está inmersa en la conducta de las personas de una organización puede traer consecuencias muy graves: "Un profesional que no es ético puede generar consecuencias negativas en la vida de otras personas. A modo de ejemplo, si se es médico, abogado o político, se puede cambiar el destino de esa persona, de la familia y de la sociedad" (Llanos, 2014). De esta manera, el comportamiento ético, constituye la piedra angular para crear cimientos sólidos en los cuales se afianzarán procesos y decisiones trasparentes que permitirán a las empresas trascender en el tiempo.

Por tanto, si el sector educativo y el gobierno focalizan sus esfuerzos en desarrollar tempranamente estas 5 habilidades blandas desde las aulas, se estaría garantizando elevar el nivel de empleabilidad de las personas, mejorar la productividad de las empresas y por ende elevar los estándares de vida de la sociedad en general.

\section{¿Cómo desarrollar competencias blandas?}

En el momento de optar por el desarrollo de habilidades blandas surgen algunos obstáculos. El primero de ellos se refiere a la percepción sobre la dificultad de definir, enseñar, y evaluar estas competencias. El segundo, a la atención que en la actualidad se está brindando a la tecnología que tiende a desvalorizar su importancia. En algunos casos enseñarlas es considerado como responsabilidad de alguien más, permitiendo que el estudiante reaccione con apatía respecto a la necesidad de contar con estas habilidades. Por otro lado, se evidencia una falta de conexión entre el ámbito de la enseñanza y el ámbito laboral en lo que se refiere a habilidades blandas. La comunidad laboral busca empleados que cuenten con estas habilidades, mientras que los educadores no les atribuyen suficiente importancia (PCBEE, 2000).

De acuerdo a Singer, Guzmán y Donoso (2009) hay poca evidencia de que exista un entrenamiento explícito en competencias blandas dentro de los procesos educativos formales. La razón puede deberse a que la evaluación de los sistemas educativos se hace en función de conocimientos y procesos cognitivos, por ejemplo los países se comparan en su rendimiento académico en ciencias, matemáticas y otras materias ${ }^{2}$. Por otra parte, el mercado laboral cada vez

\footnotetext{
1 "Enron está catalogado como el arquetipo del fraude empresarial planificado, el más grande fraude empresarial de la historia y la mayor quiebra de la historia empresarial." (Márquez, 2014). El autor señala que este fraude fue posible gracias al apoyo de "técnicas fraudulentas que estuvieron respaldadas por la prestigiosa firma auditora Arthur Andersen (AA)". En el 2001 la reputación de Enron empezó a decaer por rumores de pago de sobornos y tráfico de influencias. En ese año se reveló al público que "buena parte de las ganancias de la empresa eran resultado de negocios con una de sus subsidiarias, que le permitía "maquillar" en los balances financieros las gigantescas pérdidas sufridas" En el 2005 la empresa cayó en banca rota arrastrando a (AA) quienes fueron declarados culpables por la destrucción de documentos que evidenciaban pérdidas de más de 1,000 millones de dólares (Márquez, 2014). Los ejecutivos y administradores, cobraban gran parte del salario en acciones, lo que les motivaba a inflar y maquillar sus informes para que los beneficios aparentes fueran mayores y las acciones de la empresa aumentaran de valor... las consecuencias fueron todas negativas. Perjudicaron a sus trabajadores, inversores y acreedores, que perdieron sus trabajos, inversiones y derechos de cobro sobre la empresa respectivamente. Además, afectaron sobre las pensiones de jubilación (Martin García, 2014)

${ }^{2}$ Los autores hacen referencia a la prueba del Sistema de Medición de la Calidad de la Educación (SIMCE) en $4^{\circ}$ y $8^{\circ}$ básico, y la Prueba de Selección Universitaria (PSU), que en Chile son el principal indicador de calidad de la enseñanza y miden el nivel de conocimiento de los estudiantes. Los países también se comparan mediante tests cognitivos, tales como la prueba Program for International Student Assessment (PISA), o del Trends in International Mathematics and Science Study (TIMSS). También existe el índice de Competitividad Global que elabora el Foro Económico Mundial que mide la calidad de la educación a través: del rendimiento de los estudiantes en ciencias y
} 
brinda mayor valor a las competencias blandas antes que las habilidades duras. Mientras que éstas últimas se entrenan fácilmente, el aprendizaje de una competencia blanda puede tardar muchos años. Los autores mencionan que la dificultad de entrenar las competencias blandas se explica por su fuerte dependencia de los rasgos de personalidad.

Sin embargo, las competencias blandas pueden entrenarse desde muy temprana edad, y algunos rasgos de la personalidad pueden modificarse en función a los roles o experiencias que las personas afrontan a lo largo de sus vidas (Gottschalk, 2005; Roberts, Helson, \& Klohnen, 2002;). Singer, Guzmán \& Donoso (2009) señalan que inclusive intervenciones puntuales como asistir a talleres sobre soft skills mejoran significativamente estas habilidades, por lo que sugieren que se generen estrategias sistemáticas de mediano y largo plazo para conseguir mayores beneficios. Además, es muy importante brindar continuamente espacios de práctica, puesto que solo la repetición de lo aprendido consigue una automatización de la conducta (Driskell, Willis, \& Cooper, 1992).

Mientras que las habilidades técnicas son parte de muchos y excelentes planes de estudio, las habilidades blandas necesitan mayor énfasis en los programas universitarios para que los estudiantes aprendan la importancia de las habilidades blandas al principio de sus programas académicos antes de embarcarse en el mercado laboral (Wellington, 2005). Los educadores tienen una responsabilidad especial en lo que concierne al desarrollo de soft skills, porque durante la etapa universitaria es donde se obtiene un mayor impacto en el desarrollo de esas habilidades (Schulz, 2008). El 60\% de líderes piensan que es más fácil cultivar estas habilidades en alumnos antes que en empleados con experiencia, alegando que éstos son más abiertos a nuevas ideas, cuando se los compara con trabajadores que ya cuentan con ciertos patrones y hábitos (American Management Association, 2012).

Para la enseñanza de las habilidades blandas muchos instructores de escuelas de negocios utilizan la lectura y análisis de casos de estudio. Sin restar valor a este método, mientras más se enfatiza en tipos de aprendizaje pasivos y teóricos, antes que en la enseñanza de habilidades de liderazgo y trabajo en equipo, es realmente poco probable que los estudiantes desarrollen dichas habilidades, para hacerlo es necesario generar también oportunidades más participativas y prácticas. Un modelo de enseñanza completo debe estar compuesto por oportunidades para: primero conocer, luego hacer, y por último ser (Datar, Garvin, \& Cullen, 2010). Las habilidades blandas generalmente se desarrollan mejor a través de la participación activa de los estudiantes, se requiere práctica y retroalimentación continua para el refinamiento de habilidad (Blaszcynski \& Green, 2012).

Lo más importante para desarrollar las competencias blandas es entender que se aprenden con una pequeña cantidad de inputs altamente enfocados y relevantes, una gran cantidad de experiencia en el mundo real, la práctica dentro y fuera de la zona de confort, y la retroalimentación oportuna, pertinente y constructiva por parte de otras personas en una

matemáticas; la gestión de la academia; acceso a internet en las escuelas; disponibilidad de servicios de investigación; y, capacitación. En Ecuador, los principales indicadores de calidad de la educación están en base a estándares: a) de gestión educativa que están relacionados a procesos, b) de desempeño de los profesores, c) de aprendizaje en función de los conocimientos que los estudiantes van adquiriendo en las materias de lengua y literatura, matemáticas, estudios sociales y ciencias naturales. (República del Ecuador. Ministerio de Educación, 2012). No existe evidencia formal de un entrenamiento en habilidades blandas desde las aulas. 
comunidad de práctica, y donde las consecuencias de lo que se hace pueden ser observadas y de fácil comprensión (Chartered Institute of Personnel and Development [CIPD], 2010).

Bajo estas premisas, la enseñanza eficaz para cualquier tipo de competencia requiere primero, que el instructor demuestre o modele la habilidad o capacidad, segundo, que proporcione oportunidades para que los estudiantes practiquen la habilidad de acuerdo a los criterios de un desempeño competente, y tercero que brinde retroalimentación correctiva sobre el desempeño de los estudiantes, junto con una explicación transparente de esta retroalimentación o feedback (Bedwell, Fiore, \& Salas, 2014; Blaszcynski \& Green, 2012; Colby, Ehrlich, Sullivan, \& Dolle, 2011; Kraiger, 2003).

Kraiger (2003) señala un modelo para el aprendizaje de habilidades blandas, el mismo que se describe mediante el siguiente diagrama:

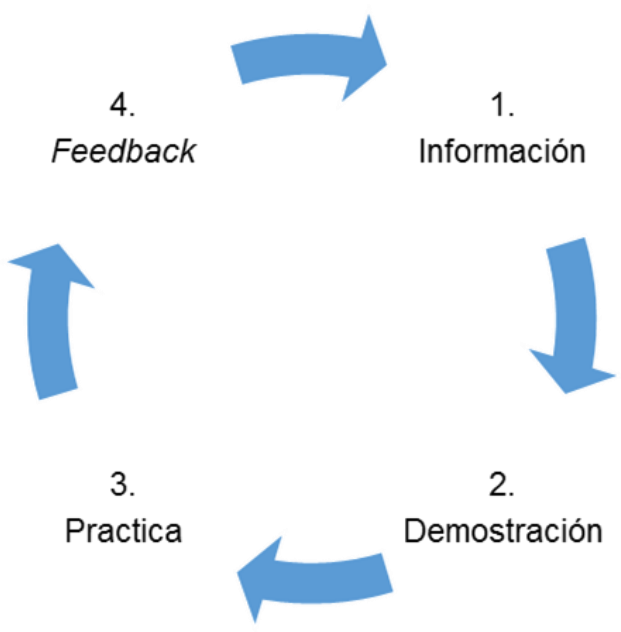

Figura 1. Diagrama sobre el modelo de aprendizaje de habilidades blandas de Kraiger.

Elaborado en función al modelo propuesto por K. Kraiger, 2003, Perspective on training and development.

El autor señala que el primer paso: información, se refiere a la presentación de los conceptos subyacentes a la habilidad que deben ser aprendidos. Demostración, es el acto de mostrar la habilidad mediante simulaciones o ambientes que asimilen situaciones del mundo real, juegos de roles, etc., en donde se demuestren las habilidades y destrezas que se deben aprender. Sin embargo, estos dos componentes no son suficientes para garantizar la eficacia de la formación. Los alumnos también deben tener la capacidad de practicar estas habilidades recién aprendidas, y deben recibir retroalimentación durante y después de todas las oportunidades de práctica (Kraiger, 2003).

Para Bedwell, Fiore, \& Salas (2014) los maestros pueden usar ciertas herramientas para cada una de estas etapas. En la fase de información, el docente debe definir claramente los objetivos de aprendizaje, y puede entregar lecturas a los alumnos en base a las habilidades que se están enseñando. En la etapa de demostración, puede hacer uso de video clips o películas en donde se ejemplifiquen estas habilidades. Para la fase de práctica, se puede solicitar a los estudiantes que desarrollen un pequeño film en base al tema que están aprendiendo donde deban demostrar esta habilidad, también se puede pedir a los alumnos que participen en ruedas de prensa simuladas, y que realicen actividades experimentales donde tengan que trabajar en equipo 
como por ejemplo, salidas al aire libre y proyectos. Con respecto a la evaluación, señalan que es importante que los docentes estén entrenados en brindar feedback efectivo y que se valgan de herramientas como cuestionarios y escalas de observación para hacer evaluaciones objetivas.

Nicol y Macfarlane-Dick (2006), describen la mejor forma de evaluar soft skills a través de las siguientes prácticas:

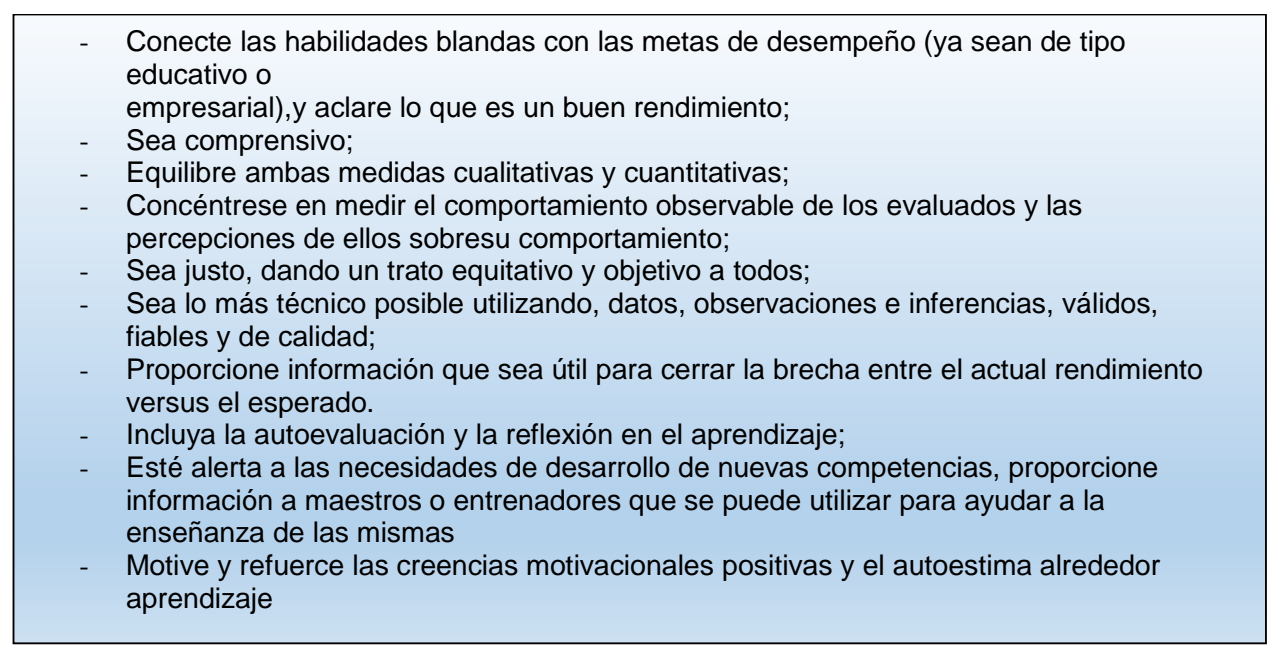

Figura 2. La mejor práctica para la práctica de la evaluación de las competencias transversales. Tomado de Nicol y Macfarlane-Dick (2006).

PCBEE (2000) en una de sus políticas enumera las estrategias necesarias para el desarrollo de habilidades blandas: 1) Modelado: incluye juegos de rol, permite proporcionar a los estudiantes ejemplos de las expectativas de comportamiento en los lugares de trabajo, 2) Portafolios de cuestionarios y evaluaciones: permiten a los estudiantes examinar su progreso hacia el desarrollo de estas conductas esperadas 3) Las simulaciones: proporcionan oportunidades para que los estudiantes experimenten situaciones similares a las que encontrarán en sus puestos de trabajo y carreras.

Los juegos son otra estrategia importante para desarrollar habilidades blandas, debido a que apoyan al desarrollo de la auto-eficacia, auto- aprendizaje y la reflexión sobre el rendimiento, además de las habilidades interpersonales como la cooperación, comunicación, y negociación, todas ellas necesarias para la resolución de problemas y construcción del liderazgo (Dondlinger, 2007;De Freitas \& Neuman, 2009). Inclusive se usan programas de juegos en línea a través de los cuales individuos que están geográficamente dispersos pueden interactuar mediante el uso de tecnología y el internet (De Freitas \& Routledge, 2013). El desarrollo de habilidades blandas, se facilita en el entorno de los juegos en línea, donde se pueden evidenciar las competencias crítcas y de liderazgo, y mayor intercomunicación y transparencia de las mértricas de evaluación (Reeves \& Malone, 2007).

Otra alternativa para la enseñanza de habilidades blandas es permitir a los estudiantes la participación en proyectos comunitarios o actividades artísticas, en donde puedan vivenciar otras realidades y palpar los resultados del trabajo realizado en equipo (Silva, 2013). 
Alles (2008) propone algunas otras iniciativas que contribuyen al desarrollo de soft skills: a) Designar a una persona de forma interina en un puesto, b) Recibir una guía experta para el desarrollo - mentor, c) Coaching

McEwen (2010) manifiesta que para enseñar habilidades blandas se debe implementar un proceso continuo a lo largo del plan de estudios en lugar de un enfoque de solo "una vez". Estas habilidades deberían integrarse regularmente en las clases y ser reforzadas constantemente.

Por otra parte, el rol del gobierno también es clave para el desarrollo de soft skills, debido a que constituye el núcleo del cual nacen las directrices que han de tomar los planes educativos para alcanzar los objetivos que se persiguen como sociedad. Estados como La Rioja en España, han hecho importantes avances en este aspecto, promoviéndose, desde el Gobierno regional, la iniciativa de trabajar en conjunto con las universidades para combatir el desempleo mediante un proyecto que busca educar en habilidades blandas para que los estudiantes sean protagonistas en su meta de mejorar sus opciones de empleabilidad. Tanto el gobierno de la Rioja como las autoridades del sector educativo, declararon la importancia que tienen las universidades como aliadas estratégicas para el desarrollo social y económico de la nación, y reconocieron que educar en habilidades blandas es un proceso vital para disminuir el desempleo (EuropaPress, 2014).

Según lo antes expuesto, si es posible desarrollar habilidades blandas realizando intervenciones puntuales sobre esta temática e incorporando herramientas como: el diseño y desarrollo de proyectos en equipo, actividades artísticas o con la comunidad, aplicando el círculo del aprendizaje de Kraiger, juegos, simulaciones, modelado, mentoring etc., El mejor momento aunque no el único para hacerlo, es durante la trayectoria universitaria debido a que este organismo tiene como misión el completar la educación formal que prepara a los estudiantes para el mercado laboral. En la vida profesional post académica y como ya se señaló, resultará mucho más complicado desarrollar estas habilidades, en tanto que se trata con empleados que ya cuentan con experiencia y hábitos.

Para conseguir un cambio significativo, la enseñanza de estas habilidades debe impartirse de forma transversal, sistemática y a largo plazo desde las aulas. Es necesaria la existencia de un proceso formal en donde se defina claramente las habilidades blandas que se esperan desarrollar, el nivel actual de los estudiantes, el nivel deseado y el alcanzado, y que continuamente exista retroalimentación y práctica para que éstas lleguen al nivel necesario que permita promover la carrera profesional de los alumnos y las posibilidades de tener éxito en el empleo. Inclusive se lo puede lograr sin necesidad de transformar la malla curricular, incluyendo en las distintas materias las actividades antes mencionadas y brindando un nivel adecuado de seguimiento.

\section{Conclusiones}

Es importante que desde las universidades se prepare a los estudiantes en habilidades blandas para que éstos egresen con herramientas que agreguen una ventaja diferenciadora que les permita competir en el mercado laboral, conseguir buenos empleos, obtener buenas remuneraciones, y en general mantener un elevado sentido de bienestar consigo mismo y en distintos ámbitos de su vida. Para la enseñanza de estas habilidades es necesario modificar los 
contenidos de la educación, confiriendo tanta importancia a los conocimientos técnicos como a las competencias blandas. Esto podría apuntalarse, mediante un proceso formal, sistemático y de largo plazo, que establezca claramente el nivel deseado de estas habilidades y el alcanzado por los estudiantes, y que propicie continuamente espacios de práctica para que los alumnos incorporen soft skills a su perfil a lo largo de su carrera.

Al entrenar futuros empleados que cuenten con los atributos esperados por las empresas, se estaría beneficiando a la sociedad en general, puesto que mayor cantidad de personas estarían en la capacidad de conseguir empleo y desarrollar al máximo sus potencialidades. Al hacerlo más allá de elevar su sentido de satisfacción y realización personal mediante el trabajo, estarían también incrementando la productividad y competitividad de las empresas, lo que a su vez alentaría un ciclo de desarrollo social y económico.

Por otro lado, se evidencia la necesidad de obtención de datos longitudinales en lo que se refiere a la enseñanza y evaluación de soft skills. Para la evaluación de habilidades duras como conocimientos, si se puede encontrar datos cuantitativos que pueden ser rastreados a través de las instituciones y en el tiempo. No ocurre esto en la evaluación y formación de las competencias transversales. También es probable que los datos sean cualitativos, por lo que incluso estando disponibles serían difícilmente analizables (Gibb, 2014). El autor indica que se requiere de pruebas estandarizadas y del diseño de criterios de referencia para el estudio de las mismas. Propone la recopilación de datos utilizando encuestas, la comparación entre distintas prácticas en la enseñanza y desarrollo de soft skills entre instituciones e inclusive países.

Finalmente, se evidencia la necesidad de que en los próximos años los gobiernos, colegios, institutos técnicos, universidades, y empresas, se conviertan en socios vitales para la construcción de un sistema de aprendizaje permanente para la vida lo que se traduce en la enseñanza de soft skills. Esto funcionará tanto para los estudiantes como para las economías locales fortaleciéndose así el vínculo entre la educación, los empleadores y las oportunidades de carrera para los estudiantes (OVAE, 2007 citado en Ellis, Kisling, \& Hackworth, 2014)

\section{Bibliografía}

Alles, M. (2007). Dirección Estratégica de Recursos Humanos: Gestión por competencias. Buenos Aires: Granica.

Alles, M. (2008). Desarollo del Talento Humano: Basado en competencias. Buenos Aires: Granica.

American Management Association. (2012). 2012 Critical Skills Survey. Obtenido de www.amanet.org: www.amanet.org

Bedwell, W. L., Fiore, S. M., \& Salas, E. (2014). Developing the Future Workforce: An Approach for Integrating Interpersonal Skills Into the MBA Classroom. Academy of Management Learning \& Education , 13(2), 171 - 186. 
Bermúdez, M., Montaño, M., \& Santillan, V. (2011). La práctica docente y el desarrollo de habilidades intelectuales en la formación profesional por competencias. Horizontes Educacionales, 16(2), 43-56.

Blanco, A. (2009). Desarrollo y evaluación de competencias en Educación Superior. Madrid: Narcea S.A. Ediciones.

Blaszcynski, C., \& Green, D. (2012). Effective Strategies and Activities for developing soft skills Part 1. Journal of applied research for Business Instruction, 10(1). 1.

Bonnie J., W., Mitchel, G. W., Skinner, L. B., \& White, B. J. (2010). Essential Soft Skills for success in the twenty - first century workforce as perceived by business educators. The Delta Pi Epsilon Journal, 52(1), 43-53.

Brungardt, C. (2011). The Intersection Between Soft Skill Development and Leadership Education. Journal of Leadership Education, 10(1), 1-22.

Carnevale, A. P., Gainer, L. J., \& Meltzer, A. S. (1990). The Essential Skills Employers Want. San Francisco: Jossey-Bass.

Chartered Institute of Personnel and Development [CIPD]. (2010). Using the head and heart at work. A business case for soft skills. London: Chartered Institute of Personnel and Development.

Cline, S. (2005). Soft skills make the difference in the workplace. Colorado Springs Business Journal. Obtenido de http://csbj.com/2005/04/01/soft-skills-make-the-difference-in-theworkplace/

Codina, A. (07 de 06 de 2005). degerencia.com. Obtenido de http://www.degerencia.com/articulo/eficacia_y_creatividad_en_la_gerencia

Colby, A., Ehrlich, T., Sullivan, W., \& Dolle, J. R. (2011). Rethinking undergraduate business education: Liberal learning for the profession. San Francisco: Jossey - Bass.

Collins Dictionary. (25 de Agosto de 2014). Soft Skills Definition. Obtenido de Collins Dictionary: http://www.collinsdictionary.com/dictionary/english/soft-skills?showCookiePolicy=true

Datar, S. M., Garvin, D. A., \& Cullen, P. G. (2010). Rethinking the MBA: Business education at a crossroads. Boston: Harvard Business Press.

De Freitas, S., \& Neuman, T. (2009). The use of "exploratory learning" for supporting immersive learning in virtual enviroments. Computers and Education, 52(2), 343-352.

De Freitas, S., \& Routledge, H. (2013). Designing leadership and soft skills in educational games: The e-leadership and soft skills educational games design model (ELESS). British Journal of Educational Technology, 44(6), 951 - 968. 
Dondlinger, M. J. (2007). Educational video games design: a review of the literature. Journal of Applied Educational Technology, 4(1), 21-31.

Driskell, J. E., Willis, R. P., \& Cooper, C. (1992). Effect of overlearning on retention. Journal of Applied Psychology, 77, 615 - 622.

Ellis, M., Kisling, E., \& Hackworth, R. G. (2014). Teaching Soft Skills Employers Need. Community College Journal of Research and Practice, 38(5), 433 - 453. doi:10.1080/10668926.2011.567143

Employers Value Communication and Interpersonal Skills. (2004). Keying In, 3(14), 1-6.

EuropaPress. (2014). Erro subraya la importancia de las habilidades blandas para mejorar las opciones de encontrar un empleo. Obtenido de lainformación.com Web site: http://noticias.lainformacion.com/mano-de-obra/mercado-laboral/erro-subraya-laimportancia-de-las-habilidades-blandas-para-mejorar-las-opciones-de-encontrar-unempleo_DUaCYo3PC9zpw7KPgpEx71/

Flores, M. (2014). Liberar el potencial humano. Latin Trade, 36-37.

Gibb, S. (2014). Soft skills assessment: theory development and the research agenda. International Journal of Lifelong Education, 33(4), 455-471.

Glenn, J. L. (2008). The "new" customer service model: Customer advocate, company ambassador. Business Education Forum, 62(4), 7-13.

Gottschalk, P. (2005). Can work alter welfare recipients beliefs? Journal of Policy Analysus and Management, 24(3), 485 - 498.

Hales, S. (2012). Skills for tomorrow. American Management Association. Washington: Special Libraries Association .

Han, L. (2014). Hard Skills vs. Soft Skills - Difference and Importance. Obtenido de Bemycareercoach.com.

Hewitt. (2006). 9 Soft Skills For Success. Obtenido de Askmen.com: http://www.askmen.com/money/career_100/121_career.html

James, R. F., \& James, M. L. (2004). Teaching career and technical skills in a "mini" business world. Business Education Forum, 59(2), 39-41.

Kraiger, K. (2003). Perspective on training and development. NJ: In W.C. Borman, D. R. Ilgen \& R. J. Klimoski (Eds.). 
Lazarus, A. (2013). Soften Up: The Importance of Soft Skills for Job Success. Physician Executive Journal, 40-45.

Llanos, L. (2014). La importancias de cultivar la ética profesional. Obtenido de www.educamericas.com: $\quad$ http://www.educamericas.com/articulos/reportajes/laimportancia-de-cultivar-la-etica-profesional

Manpower. (2013). Estudio Manpowergroup sobre escasez de talento. Barcelona: MANPOWERGROUP.

Márquez, J. (26 de enero de 2014). El mega fraude "Enron". Elnuevodiario.com. Obtenido de http://www.elnuevodiario.com.ni/opinion/308847

Martin García, L. (Mayo de 2014). Ética y Empresa: El caso Enron. Obtenido de Queaprendemoshoy.com: http://queaprendemoshoy.com/etica-y-empresa-el-caso-enron/

McEwen, B. E. (2010). Cross-cultural and international career exploration and employability skills. Cross-cultural and international business education, 2010 [NBEA) yearbook.(48), 140-159.

Mertens, L. (1996). Competencia laboral: sistema, surgimiento y modelos. Montevideo: Cinterfor/OIT.

Nicole, D. J., \& Macfarlane - Dick, D. (2006). Formative assessment and self regulated learning: A model and seven principles of good feedback practise. Studies in Higher Education(31), 199 - 218.

Perreault, H. (2004). Business educators can take a leadership role in character education. Business Education Forum, 1(59), 43-53.

Policies Commission for Business and Economic Education [PCBEE]. (2000). This we believe about teaching soft skills: Human relations, self-management, and workplace enhancement. This we believe about teaching soft skills: Human relations, selfmanagement, and workplace enhancement. Policy Statement 67. Obtenido de http://www.nbea.org/curriculum/no67.html

Puga, J., \& Martinez, L. (2008). Competencias Directivas en Escenarios Globales. Estudios Gerenciales, 24(109), 87-103.

Reeves, B., \& Malone, T. (2007). Leadership in games and at work:implications for the enterprise of massively multiplayer online role-playing games. Obtenido de www.seriosity.com: http://www.seriosity.com/ downloads/Leadership_In_Games_Seriosity_and_IBM

República del Ecuador Consejo Nacional de Planificación Rafael Correa Presidente. (2013 - 2017). Plan Nacional del Buen Vivir. 
República del Ecuador. Ministerio de Educación. (2012). Estándares de Calidad de la Educación.

Roberts, B. W., Helson, R., \& Klohnen, E. C. (2002). Personality development and growth in women across 30 years: Three perspectives. Journak of Personality, 70(1), 79-102.

Robles, M. (2012). Executive Perceptions of the Top 10 Soft Skills Needed in Today's Workplace. Business Communication Quarterly, 75(4), 453-465.

Robles, M. M. (2012). Executive Perceptions of the Top 10 Soft Skills Needed in Today's Workplace. Business Communication Quarterly, 75(4), 453-465.

Schulz, B. (2008). The Importance of Soft Skills: Education beyond academic knowledge. NAWA Journal of Language and Communicatio, 2(Issue 1), 146-154.

Silva, M. (2013). Habilidades Blandas fundamentales para el desarollo personal. Revista Educar, 22-23. Obtenido www.cide.cl/.../Revista_educar_Habilidades_blandas_MJValdebenito.pdf

Singer, M., Guzmán, R., \& Donoso, P. (15 de Enero de 2009). Entrenando Competencias Blandas en Jóvenes. Santiago de Chile, Chile. Obtenido de www.inacap.cl

Spencer, L., \& Spencer, S. (1993). Competence at work, models for superior performance. USA: John Wiley \& Sons, Inc.

Sutton, N. (2002). Why can't we all just get along? Computing Canada, 28(16), 20.

Tola, J. (2012). Las represalias de la globalización hacia el talento humano ecuatoriano. FEN Opina(12). Obtenido de http://www.fen.espol.edu.ec/tolajenny_lasrepresaliasdelaglobalizacionhaciaeltalentohuma noecuatoriano

U.S. Department of education Office of Vocational \& Adult Education (OVAE). (2007). www.ed.gov. Obtenido de Community Colleges Home: http://www.ed.gov/about/offices/list/ovae/pi/cclo/index.html\#factfigure

Vargas, F. (2006). De las virtudes laborales a las competencias clave: un nuevo concepto para antiguas demandas. Politécnica(3), 13-26.

Wellington, J. (2005). The "soft skills" of success: Be it high tech, low tech, or no tech. Vital Speeches of the Day, 71, 628.

Wilhelm, W. J. (2004). Determinants of moral reasoning: Aca- demic factors, gender, richness of life experiences, and religious preferences. The Delta Pi Epsilon Journal, XLVI(2), 105 121. 